\title{
The Optimum Quantity of Capital and Debt*
}

\author{
Marcus Hagedorn ${ }^{\dagger}$ \\ Hans Holter \\ Yikai Wang ${ }^{\S}$
}

February 15, 2015

\begin{abstract}
In this paper we consider an optimal taxation problem in an incomplete markets model to study the optimal quantity of capital and debt. The government commits itself ex-ante to a tax schedule and government debt. In contrast to most of the existing literature these instruments are chosen to to maximize agents' discounted present value of lifetime utility.

Whereas the literature mainly focuses on characterizing the steady state which maximizes welfare, we characterize and compute the optimal policy along the full transition path. In particular our characterization takes into account that the optimal long-run policy depends on capital, debt and taxation during the transition path.

We show theoretically that it is optimal to equalize the pre-tax return on capital and the rate of time preference in the long-run, i.e. the capital stock satisfies the modified golden-rule.

Quantitatively we find that the tax on capital is around 3 percent in the longrun. Labor is taxed at a much higher rate where the precise number depends on the labor supply elasticity. For standard choices for this elasticity we find a labor tax rate of almost 40 percent to be optimal in the long-run. The reason for such a hight tax rate on labor income is that labor income is risky. Taxing this risky income and redistributing it back through lump-sum transfers improves ex-ante welfare in the long-run.

Transfers and the optimal level of debt along the transition are chosen to equalize the amount of redistribution over time. Initially capital is taxed higher than in the long-run since it is inelastically supplied whereas labor is taxed less than in steady state.
\end{abstract}

\footnotetext{
${ }^{*}$ We thank seminar participants at the University of Oslo for helpful comments.

${ }^{\dagger}$ University of Oslo, Department of Economics, Box 1095 Blindern, 0317 Oslo, Norway. Email: marcus.hagedorn07@gmail.com

${ }^{\ddagger}$ University of Oslo, Department of Economics, Box 1095 Blindern, 0317 Oslo, Norway. Email:

$\S$ University of Oslo, Department of Economics, Box 1095 Blindern, 0317 Oslo, Norway. Email:.
} 
Keywords: Optimal Debt, Incomplete Markets, Capital Taxation

JEL codes: 


\section{Introduction}

In this paper we consider an optimal taxation problem in an incomplete markets model to study the optimal quantity of capital and debt. The government commits itself ex-ante to a tax schedule and government debt. In contrast to most of the existing literature these instruments are chosen to to maximize agents' discounted present value of lifetime utility.

Whereas the literature mainly focuses on characterizing the steady state which maximizes welfare, we characterize and compute the optimal policy along the full transition path. In particular our characterization takes into account that the optimal long-run policy depends on capital, debt and taxation during the transition path.

We show theoretically that it is optimal to equalize the pre-tax return on capital and the rate of time preference in the long-run, i.e. the capital stock satisfies the modified golden-rule.

Quantitatively we find that the tax on capital is around 3 percent in the long-run. Labor is taxed at a much higher rate where the precise number depends on the labor supply elasticity. For standard choices for this elasticity we find a labor tax rate of almost 40 percent to be optimal in the long-run. The reason for such a hight tax rate on labor income is that labor income is risky. Taxing this risky income and redistributing it back through lump-sum transfers improves ex-ante welfare in the long-run.

Transfers and the optimal level of debt along the transition are chosen to equalize the amount of redistribution over time. Initially capital is taxed higher than in the long-run since it is inelastically supplied whereas labor is taxed less than in steady state.

\section{The Model}

\subsection{Households' Problem}

Following Acikgoz 2014, we formulate a Household's problem as:

$$
V^{H}\left(a_{0} ; \bar{r}, \bar{w}\right)=\max _{\left\{a_{t+1}\left(h^{t}\right), c_{t}\left(h^{t}\right)\right\}} \sum_{t=0}^{\infty} \beta^{t} \sum_{h^{t}} \Pi\left(h^{t}\right) u\left(c_{t}\left(h^{t}\right), n_{t}\left(h^{t}\right)\right)
$$

subject to

$$
\begin{aligned}
c_{t}\left(h^{t}\right)+a_{t+1}\left(h^{t}\right) & \leq a_{t}\left(h^{t-1}\right)\left(1+\bar{r}_{t}\right)+\bar{w}_{t} e_{t}\left(h^{t}\right) n_{t}\left(h^{t}\right)+T_{t}, \\
a_{t+1}\left(h^{t}\right) & \geq-\underline{a} .
\end{aligned}
$$


We use the utility function $u(c, n)=\frac{c^{1-\sigma}}{1-\sigma}-\chi \frac{n^{1+\phi}}{1+\phi}$, so a household's labor supply can be expressed as

$$
\begin{aligned}
u_{c}\left(c_{t}, n_{t}\right) e_{t} \bar{w}_{t}+u_{n}\left(c_{t}, n_{t}\right) & =0 \Rightarrow \\
\frac{-u_{n}\left(c_{t}, n_{t}\right)}{u_{c}\left(c_{t}, n_{t}\right)} & =e_{t} \bar{w}_{t} \Rightarrow \\
\frac{\chi n_{t}^{\phi}}{c_{t}^{-\sigma}} & =e_{t} \bar{w}_{t} \Rightarrow \\
n_{t} & =\left(\chi^{-1} e_{t} \bar{w}_{t} c_{t}^{-\sigma}\right)^{\frac{1}{\phi}}, \\
y_{t} & =\left(\chi^{-1} e_{t}^{1+\phi} \bar{w}_{t}^{1+\phi} c_{t}^{-\sigma}\right)^{\frac{1}{\phi}} .
\end{aligned}
$$

Moreover,

$$
e_{t} w_{t} u_{c t}+u_{n t}=0
$$

will be a useful expression to simplify expressions later. Keeping the expressions of $n$ and $y$ in mind, the households' policy functions solve the following system of necessary conditions

$$
\begin{aligned}
u^{\prime}\left(c_{t}\left(h^{t}\right)\right) & \geq \beta\left(1+\bar{r}_{t+1}\right) \sum_{h^{t+1}} \Pi\left(h^{t+1} \mid h^{t}\right) u^{\prime}\left(c_{t+1}\left(h^{t+1}\right)\right), \\
0= & \left(a_{t+1}\left(h^{t}\right)+\underline{a}\right)\left(u^{\prime}\left(c_{t}\left(h^{t}\right)\right)-\beta\left(1+\bar{r}_{t+1}\right) \sum_{h^{t+1}} \Pi\left(h^{t+1} \mid h^{t}\right) u^{\prime}\left(c_{t+1}\left(h^{t+1}\right)\right)\right) \\
c_{t}\left(h^{t}\right)+a_{t+1}\left(h^{t}\right) & \leq a_{t}\left(h^{t-1}\right)\left(1+\bar{r}_{t}\right)+y_{t} \\
a_{t+1}\left(h^{t}\right)+\underline{a} & \geq 0 .
\end{aligned}
$$

\subsection{Ramsey Problem}

The Ramsey problem in Aiyagari (1994) can be formed as

$$
V\left(a_{0}, B_{0}\right)=\max _{\left\{\bar{r}_{t}, \bar{w}_{t}, B_{t+1}, T_{t}, a_{t+1}\left(h^{t}\right), c_{t}\left(h^{t}\right)\right\}} \sum_{t=0}^{\infty} \beta^{t} \sum_{h^{t}} \Pi\left(h^{t}\right) u\left(c_{t}\left(h^{t}\right), n_{t}\left(h^{t}\right)\right)
$$


subject to

$$
\begin{aligned}
c_{t}\left(h^{t}\right)+a_{t+1}\left(h^{t}\right) & \leq a_{t}\left(h^{t-1}\right)\left(1+\bar{r}_{t}\right)+y_{t}\left(h^{t}\right)+T_{t} \\
u^{\prime}\left(c_{t}\left(h^{t}\right)\right) & \geq \beta\left(1+\bar{r}_{t+1}\right) \sum_{h^{t+1}} \Pi\left(h^{t+1} \mid h^{t}\right) u^{\prime}\left(c_{t+1}\left(h^{t+1}\right)\right) \\
0= & \left(a_{t+1}\left(h^{t}\right)+\underline{a}\right) \\
& \left(u^{\prime}\left(c_{t}\left(h^{t}\right)\right)-\beta\left(1+\bar{r}_{t+1}\right) \sum_{h^{t+1}} \Pi\left(h^{t+1} \mid h^{t}\right) u^{\prime}\left(c_{t+1}\left(h^{t+1}\right)\right)\right) \\
G_{t}+T_{t}+\left(1+\bar{r}_{t}\right) B_{t}+\bar{r}_{t} K_{t}+\bar{w}_{t} N_{t} & \leq F\left(K_{t}, N_{t}\right)+B_{t+1}, \\
K_{t+1} & =\sum_{h^{t}} \Pi\left(h^{t}\right) a_{t+1}\left(h^{t}\right)-B_{t+1} \\
N_{t} & =\sum_{h^{t}} \Pi\left(h^{t}\right) e_{t} n_{t}\left(h^{t}, \bar{w}_{t}, c_{t}\right) .
\end{aligned}
$$

Let $\beta^{t} \Pi\left(h^{t}\right) \theta_{t+1}\left(h^{t}\right)$ and $\beta^{t} \Pi\left(h^{t}\right) \eta_{t+1}\left(h^{t}\right)$ represent the Lagrange multipliers for (1) and (3) respectively, and define $\lambda_{t+1}\left(h^{t}\right) \equiv \eta_{t+1}\left(h^{t}\right)\left(a_{t+1}\left(h^{t}\right)+\underline{a}\right)-\theta_{t+1}\left(h^{t}\right)$, then we can write down the Lagrangian as

$$
\begin{aligned}
L & =\sum_{t=0}^{\infty} \beta^{t} \sum_{h^{t}} \Pi\left(h^{t}\right) u\left(c_{t}\left(h^{t}\right)\right) \\
& +\sum_{t=0}^{\infty} \beta^{t} \sum_{h^{t}} \Pi\left(h^{t}\right) \theta_{t+1}\left(h^{t}\right)\left(u^{\prime}\left(c_{t}\left(h^{t}\right)\right)-\beta\left(1+\bar{r}_{t+1}\right) \sum_{h^{t+1}} \Pi\left(h^{t+1} \mid h^{t}\right) u^{\prime}\left(c_{t+1}\left(h^{t+1}\right)\right)\right) \\
& -\sum_{t=0}^{\infty} \beta^{t} \sum_{h^{t}} \Pi\left(h^{t}\right) \eta_{t+1}\left(h^{t}\right)\left(a_{t+1}\left(h^{t}\right)+\underline{a}\right) \\
& \left(u^{\prime}\left(c_{t}\left(h^{t}\right)\right)-\beta\left(1+\bar{r}_{t+1}\right) \sum_{h^{t+1}} \Pi\left(h^{t+1} \mid h^{t}\right) u^{\prime}\left(c_{t+1}\left(h^{t+1}\right)\right)\right) \\
& =\sum_{t=0}^{\infty} \beta^{t} \sum_{h^{t}} \Pi\left(h^{t}\right)\left[u\left(c_{t}\left(h^{t}\right)\right)+u^{\prime}\left(c_{t}\left(h^{t}\right)\right)\right. \\
& {\left.\left[\theta_{t+1}\left(h^{t}\right)-\theta_{t}\left(h^{t-1}\right)\left(1+\bar{r}_{t}\right)-\eta_{t+1}\left(h^{t}\right)\left(a_{t+1}\left(h^{t}\right)+\underline{a}\right)+\eta_{t}\left(h^{t-1}\right)\left(a_{t}\left(h^{t-1}\right)+\underline{a}\right)\left(1+\bar{r}_{t}\right)\right]\right] } \\
& =\sum_{t=0}^{\infty} \beta^{t} \sum_{h^{t}} \Pi\left(h^{t}\right)\left[u\left(c_{t}\left(h^{t}\right)\right)+u^{\prime}\left(c_{t}\left(h^{t}\right)\right)\left[\lambda_{t}\left(h^{t-1}\right)\left(1+\bar{r}_{t}\right)-\lambda_{t+1}\left(h^{t}\right)\right]\right]
\end{aligned}
$$


subject to

$$
\begin{aligned}
c_{t}\left(h^{t}\right)+a_{t+1}\left(h^{t}\right) & \leq a_{t}\left(h^{t-1}\right)\left(1+\bar{r}_{t}\right)+y\left(h^{t}, \bar{w}_{t}, c_{t}\right)+T_{t}, \\
a_{t+1}\left(h^{t}\right)+\underline{a} & \geq 0 \\
G_{t}+T_{t}+\left(1+\bar{r}_{t}\right) B_{t}+\bar{r}_{t} K_{t}+\bar{w}_{t} N_{t} & \leq F\left(K_{t}, N_{t}\right)+B_{t+1}, \\
K_{t+1} & =\sum_{h^{t}} \Pi\left(h^{t}\right) a_{t+1}\left(h^{t}\right)-B_{t+1}, \\
N_{t} & =\sum_{h^{t}} \Pi\left(h^{t}\right) e_{t} n_{t}\left(h^{t}, \bar{w}_{t}\right) .
\end{aligned}
$$

given the known forms of $y\left(h^{t}\right)$ and $n\left(h^{t}\right)$, with initial conditions $a_{0}\left(h^{-1}\right)=a_{0}, B_{0}$ and $\lambda_{0}\left(h^{-1}\right)=0$.

\subsection{Recursive Form}

To write the problem in the recursive form, follow Marcet (2011) to expand the state space to include Lagrange multipliers of the dynamic implementability constraints to recover stationarity. Index all households by $(s, e) \equiv(a, \lambda, e)$ and denote $\mu$ as the corresponding probability measure. Then we have $V\left(a_{0}, B_{0}\right)=W\left(\mu_{0}, B_{0}\right)$ which solves:

$W(\mu, B)=\min _{\theta^{\prime}(.), \eta^{\prime}(.) \geq 0} \max _{\bar{r}, \bar{w}, T, B^{\prime}, a^{\prime}(.), c(.)} \sum_{e} \int u(c(),. n())+.u_{c}(c()).\left[\lambda(1+\bar{r})-\lambda^{\prime}().\right] \mu(d s, e)+\beta W\left(\mu^{\prime}, B^{\prime}\right)$

subject to

$$
\begin{aligned}
c(.)+a^{\prime}(.) & \leq a(1+\bar{r})+y(.)+T \\
a^{\prime}+\underline{a} & \geq 0 \\
G+T+(1+\bar{r}) B+\bar{r} K+\bar{w} N & \leq F(K, N)+B^{\prime} \\
K & =\sum_{e} \int a \mu(d s, e)-B \\
N & =\sum_{e} \int e n(.) \mu(d s, e)=\sum_{e} \pi_{e} e n(e, \bar{w}) \\
\mu^{\prime}\left(S^{\prime}, e^{\prime}\right) & =\sum_{e} \pi_{e e^{\prime}} \int I\left[\left(a^{\prime}(.), \lambda^{\prime}(.)\right) \in S^{\prime}\right] \mu(d s, e) \\
\lambda^{\prime}(.) & =\eta^{\prime}(.)\left(a^{\prime}(.)+\underline{a}\right)-\theta^{\prime} .
\end{aligned}
$$




\subsection{Interior Solution}

Denote the multiplier for government budget constraint as $\gamma$. Then we treat $c$ as a function of other control variables, which requires substituting the expressions of $n$ and $y$ :

$$
\begin{aligned}
& c+a^{\prime}=a(1+\bar{r})+y+T \Rightarrow \\
& c+a^{\prime}=a(1+\bar{r})+\left(\chi^{-1} \bar{w}^{1+\phi} e^{1+\phi} c^{-\sigma}\right)^{\frac{1}{\phi}}+T .
\end{aligned}
$$

We can get the following useful expressions on how $n$ and $y$ respond to $c$, which of course respond to other choice variable $x \in\left\{T, B^{\prime}, \bar{r}, \bar{w}\right\}$,

$$
\begin{aligned}
& \frac{\partial n}{\partial c}=-\frac{\sigma}{\phi}\left(\chi^{-1} \bar{w} e\right)^{\frac{1}{\phi}} c^{-\frac{\sigma}{\phi}-1} \\
& =-\frac{\sigma}{\phi} \frac{n}{c}, \\
& \frac{\partial n}{\partial \bar{w}}=\frac{1}{\phi} \frac{n}{\bar{w}}, \\
& \frac{\partial y}{\partial c}=-\frac{\sigma}{\phi}\left(\chi^{-1} \bar{w}_{t}^{1+\phi} e_{t}^{1+\phi}\right)^{\frac{1}{\phi}} c_{t}^{-\frac{\sigma}{\phi}-1} \\
& =-\frac{\sigma}{\phi} \frac{y}{c} \\
& =-\frac{\sigma}{\phi} \frac{n}{c} e w \\
& =e w \frac{\partial n}{\partial c}, \\
& \frac{\partial c}{\partial T}=e \bar{w} \frac{\partial n}{\partial c} \frac{\partial c}{\partial T}+1 \Rightarrow \\
& \frac{\partial c}{\partial T}\left(1-e \bar{w} \frac{\partial n}{\partial c}\right)=1, \\
& \frac{\partial u(c, n)}{\partial T}=u_{c} \frac{\partial c}{\partial T}+u_{n} \frac{\partial n}{\partial c} \frac{\partial c}{\partial T} \\
& =u_{c} \frac{\partial c}{\partial T}\left(1+\frac{u_{n}}{u_{c}} \frac{\partial n}{\partial c}\right) \\
& =u_{c} \frac{\partial c}{\partial T}\left(1-e \bar{w} \frac{\partial n}{\partial c}\right) \\
& =u_{c} \text {. }
\end{aligned}
$$


This is essentially the envelop theorem. Similarly, we can also show that

$$
\begin{aligned}
& \frac{\partial u(c, n)}{\partial a^{\prime}}=u_{c}, \\
& \frac{\partial u(c, n)}{\partial \bar{r}}=a u_{c}, \\
& \frac{\partial u(c, n)}{\partial \bar{w}}=e n u_{c}, \\
& \frac{\partial u(c, n)}{\partial a}=(1+\bar{r}) u_{c},
\end{aligned}
$$

It is not possible to directly solve for the expression of $c$, but we can derive the necessary partial derivatives to know how $c$ responds to other control variables, including $T, a^{\prime}, \bar{r}, \bar{w}, a$, 
as follows:

$$
\begin{aligned}
& \frac{\partial c}{\partial T}=\frac{\partial y}{\partial c} \frac{\partial c}{\partial T}+1 \Rightarrow \\
& \frac{\partial c}{\partial T}=\frac{1}{1-\frac{\partial y}{\partial c}} \\
& =\frac{1}{1+\frac{\sigma}{\phi} \frac{y}{c}} \\
& \frac{\partial c}{\partial a^{\prime}}+1=\frac{\partial y}{\partial c} \frac{\partial c}{\partial a^{\prime}} \Rightarrow \\
& \frac{\partial c}{\partial a^{\prime}}=\frac{1}{\frac{\partial y}{\partial c}-1} \\
& =\frac{1}{\frac{\sigma}{\phi} \frac{y}{c}-1} \\
& \frac{\partial c}{\partial \bar{w}}=e n+e \bar{w} \frac{\partial n}{\partial \bar{w}}+e \bar{w} \frac{\partial n}{\partial c} \frac{\partial c}{\partial \bar{w}} \Rightarrow \\
& \frac{\partial c}{\partial \bar{w}}=e n+e \bar{w} \frac{1}{\phi} \frac{n}{\bar{w}}+e \bar{w}\left(-\frac{\sigma}{\phi} \frac{n}{c}\right) \frac{\partial c}{\partial \bar{w}} \Rightarrow \\
& \frac{\partial c}{\partial \bar{w}}=\frac{1+\phi}{\phi} \frac{e n}{1-\frac{\sigma}{\phi} e \bar{w} n c^{-1}}, \\
& \frac{\partial c}{\partial \bar{r}}=a+\frac{\partial y}{\partial c} \frac{\partial c}{\partial \bar{r}} \Rightarrow \\
& \frac{\partial c}{\partial \bar{r}}=\frac{a}{1-\frac{\partial y}{\partial c}} \\
& =\frac{a}{1+\frac{\sigma}{\phi} \frac{y}{c}}, \\
& \frac{\partial c}{\partial a}=(1+\bar{r})+\frac{\partial y}{\partial c} \frac{\partial c}{\partial a} \Rightarrow \\
& \frac{\partial c}{\partial a}=\frac{1+\bar{r}}{1-\frac{\partial y}{\partial c}} \\
& =\frac{1+\bar{r}}{1+\frac{\sigma}{\phi} \frac{y}{c}} .
\end{aligned}
$$


Then the interior solution to the Ramsey problem satisfies the following conditions.

$$
\begin{aligned}
\partial \lambda^{\prime}: u_{c}(c) & \geq \beta\left(1+\bar{r}^{\prime}\right) \mathbb{E}\left[u_{c}\left(c^{\prime}\right) \mid e\right] \text { with equality if } a^{\prime}>-\underline{a} \\
\partial a^{\prime}: u_{c}(c)+\frac{\partial c}{\partial a^{\prime}} u_{c c}(c)\left[\lambda(1+\bar{r})-\lambda^{\prime}\right] & =\beta \mathbb{E}\left[\left(1+\bar{r}^{\prime}\right) u_{c}\left(c^{\prime}\right)+\frac{\partial c^{\prime}}{\partial a^{\prime}} u_{c c}\left(c^{\prime}\right)\left[\lambda^{\prime}\left(1+\bar{r}^{\prime}\right)-\lambda^{\prime \prime}\right] \mid e\right] \\
& +\beta \gamma^{\prime}\left(F_{K}\left(K^{\prime}, N^{\prime}\right)-\bar{r}^{\prime}\right) \text { if } a^{\prime}>-\underline{a}, \text { otherwise } \lambda^{\prime}=0 \\
\partial T: \gamma & =\sum_{e} \int\left(u_{c}(c)+\frac{\partial c}{\partial T} u_{c c}(c)\left[\lambda(1+\bar{r})-\lambda^{\prime}\right]\right) \mu(d s, e) \\
& +\gamma\left(F_{N}(K, N)-\bar{w}\right) \frac{\partial N}{\partial T} \\
\partial B^{\prime}: \gamma & =\beta\left(1+F_{K}\left(K^{\prime}, N^{\prime}\right)\right) \gamma^{\prime} \\
\partial \bar{r}: \gamma A & =\sum_{e} \int u_{c}(c) \lambda \mu(d s, e) \\
& +\gamma\left(F_{N}(K, N)-\bar{w}\right) \frac{\partial N}{\partial \bar{r}} \\
& +\sum_{e} \int a u_{c}(c)+\frac{\partial c}{\partial \bar{r}} u_{c c}(c)\left[\lambda(1+\bar{r})-\lambda^{\prime}\right] \mu(d s, e) \\
\partial \bar{w}: \gamma N & =\gamma\left(F_{N}(K, N)-\bar{w}\right) \frac{\partial N}{\partial \bar{w}} \\
& +\sum_{e} \int e n(e, \bar{w}, c) u_{c}(c)+\frac{\partial c}{\partial \bar{w}} u_{c c}(c)\left[\lambda(1+\bar{r})-\lambda^{\prime}\right] \mu(d s, e),
\end{aligned}
$$

where

$$
\begin{aligned}
& \frac{\partial N}{\partial \bar{w}}=\sum_{e} \int\left(\frac{\partial n}{\partial \bar{w}}+\frac{\partial n}{\partial c} \frac{\partial c}{\partial \bar{w}}\right) \mu(d s, e), \\
& \frac{\partial N}{\partial T}=\sum_{e} \int \frac{\partial n}{\partial c} \frac{\partial c}{\partial T} \mu(d s, e) \\
& \frac{\partial N}{\partial \bar{r}}=\sum_{e} \int \frac{\partial n}{\partial c} \frac{\partial c}{\partial \bar{r}} \mu(d s, e) .
\end{aligned}
$$

Here the unknowns are $\lambda^{\prime}(),. a^{\prime}(),. B^{\prime}, T, \bar{r}, \bar{w}$, while $c(.) \mu(), K, N,$.$A are considered as$ functions of these unknowns, from the household and the government budget constraint, the market clearing conditions and so on. We denote the p.d.f of the distribution of $(a, \lambda, e)$ as $p(a, \lambda, e)$, while its probalistic measure is $\mu$. Similarly, we denote the p.d.f. of the distribution of $(a, e)$ as $m(a, e)$. Moreover, as we know that $c_{t}$ and $a_{t+1}$ should only depend on $\left(a_{t}, e_{t}\right)$ but not $\lambda_{t}$ because they should satisfy the households' optimization which doesn't consider $\lambda_{t}$, we can use $g_{a, t+1}\left(a_{t}, e_{t}\right)$ as the policy function of $a_{t+1}, g_{c, t}\left(a_{t}, e_{t}\right)$ the policy function of $c_{t}$, and $g_{\lambda, t+1}\left(a_{t}, \lambda_{t}, e_{t}\right)$ the policy function of $\lambda_{t+1}$. Now all the unknowns and their related 
equations can be written as follows.

$$
\begin{aligned}
u_{c}\left(c_{t}\right) & \geq \beta\left(1+\bar{r}_{t+1}\right) \mathbb{E}\left[u_{c}\left(c_{t}\right) \mid e_{t+1}\right] \text { with equality if } a_{t+1}>-\underline{a} \\
& u_{c}\left(c_{t}\right)+\frac{\partial c_{t}}{\partial a_{t+1}} u_{c c}\left(c_{t}\right)\left[\lambda_{t}\left(1+\bar{r}_{t}\right)-\lambda_{t+1}\right] \\
& =\beta \mathbb{E}\left[\left(1+\bar{r}_{t+1}\right) u_{c}\left(c_{t+1}\right)+\frac{\partial c_{t}}{\partial a_{t+1}} u_{c c}\left(c_{t+1}\right)\left[\lambda_{t+1}\left(1+\bar{r}_{t+1}\right)-\lambda_{t+2}\right] \mid e_{t+1}\right] \\
& +\beta \gamma_{t+1}\left(F_{K}\left(K_{t+1}, N_{t+1}\right)-\bar{r}_{t+1}\right) \text { if } a_{t+1}>-\underline{a}, \text { otherwise } \lambda_{t+1}=0 \\
\gamma_{t} & =\beta\left(1+F_{K}\left(K_{t+1}, N_{t+1}\right)\right) \gamma_{t+1} \\
\gamma_{t} & =\sum_{e} \iint\left(u_{c}\left(c_{t}\right)+\frac{\partial c_{t}}{\partial T_{t}} u_{c c}\left(c_{t}\right)\left[\lambda_{t}\left(1+\bar{r}_{t}\right)-\lambda_{t+1}\right]\right) p\left(a_{t}, \lambda_{t}, e_{t}\right) d a_{t} d \lambda_{t} \\
& +\gamma_{t}\left(F_{N}\left(K_{t}, N_{t}\right)-\bar{w}_{t}\right) \frac{\partial N_{t}}{\partial T_{t}}, \\
\gamma_{t} A_{t} & =\sum_{e_{t}} \iint u_{c}\left(c_{t}\right) \lambda_{t} p_{t}\left(a_{t}, \lambda_{t}, e_{t}\right) d a_{t} d \lambda_{t} \\
& +\gamma_{t}\left(F_{N}\left(K_{t}, N_{t}\right)-\bar{w}_{t}\right) \frac{\partial N_{t}}{\partial \bar{r}_{t}} \\
& +\sum_{e_{t}} \iint a_{t}\left(u_{c}\left(c_{t}\right)+u_{c c}\left(c_{t}\right)\left[\lambda_{t}\left(1+\bar{r}_{t}\right)-\lambda_{t+1}\right]\right) p\left(a_{t}, \lambda_{t}, e_{t}\right) d a_{t} d \lambda_{t} \\
\gamma_{t} N_{t} & =\gamma_{t}\left(F_{N}\left(K_{t}, N_{t}\right)-\bar{w}_{t}\right) \frac{\partial N_{t}}{\partial \bar{w}_{t}} \\
& +\sum_{e_{t}} \iint e_{t} n\left(e_{t}, \bar{w}_{t}\right)\left(u^{\prime}\left(c_{t}\right)+u^{\prime \prime}\left(c_{t}\right)\left[\lambda_{t}\left(1+\bar{r}_{t}\right)-\lambda_{t+1}\right]\right) \\
p_{t} & \left(a_{t}, \lambda_{t}, e_{t}\right) d a_{t} d \lambda_{t}
\end{aligned}
$$


subject to

$$
\begin{aligned}
c_{t}+a_{t+1} & =a_{t}\left(1+\bar{r}_{t}\right)+y\left(e_{t}, \bar{w}_{t}\right) \\
G_{t}+\left(1+\bar{r}_{t}\right) B_{t}+\bar{r}_{t} K_{t}+\bar{w}_{t} N_{t} & \leq F\left(K_{t}, N_{t}\right)+B_{t+1} \\
K_{t} & =A_{t}-B_{t} \\
A_{t} & =\sum_{e_{t}} \iint a_{t} p_{t}\left(a_{t}, \lambda_{t}, e_{t}\right) d a_{t} d \lambda_{t} \\
N_{t} & =\sum_{e_{t}} \pi\left(e_{t}\right) e_{t} n\left(e_{t}, \bar{w}_{t}\right) \\
p_{t+1}\left(a_{t+1}, \lambda_{t+1}, e_{t+1}\right) & =\sum_{e_{t}} \pi\left(e_{t+1} \mid e_{t}\right) \iint I\left[g_{a, t+1}\left(a_{t}, e_{t}\right)=a_{t+1}, g_{\lambda, t+1}\left(a_{t}, \lambda_{t}, e_{t}\right)=\lambda_{t+1}\right] \\
p_{t} & \left(a_{t}, \lambda_{t}, e_{t}\right) d a_{t} d \lambda_{t} \\
m_{t+1}\left(a_{t+1}, e_{t+1}\right) & =\sum_{e_{t}} \pi\left(e_{t+1} \mid e_{t}\right) \int I\left[g_{a, t+1}\left(a_{t}, e_{t}\right)=a_{t+1}\right] m_{t}\left(a_{t}, e_{t}\right) d a_{t} \\
\frac{\partial N_{t}}{\partial T_{t}} & =\sum_{e} \iint \frac{\partial n_{t}}{\partial c_{t}} \frac{\partial c_{t}}{\partial T_{t}} p_{t}\left(a_{t}, \lambda_{t}, e_{t}\right) d a_{t} d \lambda_{t} \\
\frac{\partial N_{t}}{\partial \bar{r}_{t}} & =\sum_{e} \iint \frac{\partial n_{t}}{\partial c_{t}} \frac{\partial c_{t}}{\partial \bar{r}_{t}} p_{t}\left(a_{t}, \lambda_{t}, e_{t}\right) d a_{t} d \lambda_{t} \\
\frac{\partial N_{t}}{\partial \bar{w}_{t}} & =\sum_{e} \iint\left(\frac{\partial n_{t}}{\partial \bar{w}_{t}}+\frac{\partial n_{t}}{\partial c_{t}} \frac{\partial c_{t}}{\partial \bar{w}_{t}}\right) p_{t}\left(a_{t}, \lambda_{t}, e_{t}\right) d a_{t} d \lambda_{t} .
\end{aligned}
$$

Now there are 12 sets of unknowns $g_{a, t+1}\left(a_{t}, e_{t}\right), g_{\lambda, t+1}\left(a_{t}, \lambda_{t}, e_{t}\right), g_{c, t}\left(a_{t}, e_{t}\right), p_{t}\left(a_{t}, \lambda_{t}, e_{t}.\right), m_{t}\left(a_{t}, e_{t}\right)$ and $\gamma_{t}, \bar{r}_{t}, \bar{w}_{t}, K_{t}, N_{t}, A_{t}, B_{t}, T_{t}$. We have 12 sets of equations to solve them.

\section{The Algorithm}

\subsection{Steady State}

Here we illustrate the algorithm to compute the steady state using a simplified example as in Aiyagari (1994) and Acikgoz (2014) where the labor supply depends on the tradeoff between the market wage and home production. In the steady state, we can use the knowledge that all the variables $\bar{r}, \bar{w}, \gamma, K, N, B$ are constant and all the policy functions do not change over 
time, to simplify the set of equations that we need to solve as follows:

$$
\begin{aligned}
u^{\prime}(c) & \geq \beta(1+\bar{r}) \mathbb{E}\left[u^{\prime}\left(c^{\prime}\right) \mid e\right] \text { with equality if } a^{\prime}>-\underline{a} \\
u^{\prime}(c)+u^{\prime \prime}(c)\left[\lambda(1+\bar{r})-\lambda^{\prime}\right] & =\beta(1+\bar{r}) \mathbb{E}\left[u^{\prime}\left(c^{\prime}\right)+u^{\prime \prime}\left(c^{\prime}\right)\left[\lambda^{\prime}\left(1+\bar{r}^{\prime}\right)-\lambda^{\prime \prime}\right] \mid e\right] \\
& +\beta \gamma\left(F_{K}(K, N)-\bar{r}\right) \text { if } a^{\prime}>-\underline{a}, \text { otherwise } \lambda^{\prime}=0 \\
1 & =\beta\left(1+F_{K}(K, N)\right) \\
\gamma & =\sum_{e} \int\left(u^{\prime}(c)+u^{\prime \prime}(c)\left[\lambda(1+\bar{r})-\lambda^{\prime}\right]\right) \mu(d s, e) \\
\gamma A & =\sum_{e} \int u^{\prime}(c) \lambda \mu(d s, e) \\
& +\sum_{e} \int a\left(u^{\prime}(c)+u^{\prime \prime}(c)\left[\lambda(1+\bar{r})-\lambda^{\prime}\right]\right) \mu(d s, e) \\
\gamma N & =\gamma\left(F_{N}(K, N)-\bar{w}\right) N^{\prime}(\bar{w}) \\
& +\sum_{e} \int e n(e, \bar{w})\left(u^{\prime}(c)+u^{\prime \prime}(c)\left[\lambda(1+\bar{r})-\lambda^{\prime}\right]\right) \mu(d s, e) \\
c+a^{\prime} & =a(1+\bar{r})+y(e, \bar{w})+T \\
\bar{w} N & \leq F(K, N) \\
K & =A-B \\
N & =\sum_{e} \pi_{e} e n(e, \bar{w}) \\
A & =\sum_{e} \int a \mu(d s, e) \\
\left.a^{\prime}, \lambda^{\prime}, e^{\prime}\right) & =\sum_{e} \pi_{e e^{\prime}} \int I\left[g_{a^{\prime}}(a, e)=a^{\prime}, g_{\lambda^{\prime}}(a, \lambda, e)=\lambda^{\prime}\right] p(a, \lambda, e) d a d \lambda \\
\left.T+\bar{r} B+e^{\prime}\right) & =\sum_{e} \pi_{e e^{\prime}} \int I\left[g_{a^{\prime}}(a, e)=a^{\prime}\right] m(a, e) d a .
\end{aligned}
$$

\section{Guess $T$.}

2. Guess $\bar{w}$. Solve for $\bar{r}(w)$ following Aiyagari (1994)

(a) Solve for $K$ from (24).

(b) Guess $\bar{r}$ and solve the household's problem: solve for $c=g_{c}(a, e), a^{\prime}=g_{a^{\prime}}(a, e)$ from (22) and (28), keeping in mind that $n=\left(\chi^{-1} \bar{w} e c^{-\sigma}\right)^{\frac{1}{\phi}}, y=\left(\chi^{-1} \bar{w}^{1+\phi} e^{1+\phi} c^{-\sigma}\right)^{\frac{1}{\phi}}$.

(c) Compute $N$ from (31). 
(d) Solve for $m(a, e)$ or equivalently $m(., e)$ from (34).

(e) Solve for $A$ from (32).

(f) Solve for $B$ from (30).

(g) Verify $\bar{r}$ using (29). If the equation is not satisfied, update $\bar{r}$.

3. Define $q \equiv \lambda / \gamma$, and solve for $q^{\prime}=g_{q^{\prime}}(a, q, e)$ from $(23)$.

(a) Approach 1. Guess $q^{\prime}(a, q, e)=g_{q^{\prime}}^{0}(a, q, e)$, and then use equation 23 to find the new $q^{\prime}(a, q, e)=g_{q^{\prime}}^{1}(a, q, e)$.

(b) Approach 2. Guess $q^{\prime}=g_{q^{\prime}}(a, q, e)=\alpha_{0}(a, e) q+\frac{\alpha_{1}(a, e)}{(1+\bar{r}) u^{\prime \prime}(c)}$ and solve for $\alpha_{0}(a, e)$ and $\alpha_{1}(a, e)$, as in Acikgoz (2014).

4. Solve for $\gamma$ from $(26)$

5. Check whether (27) is satisfied.

(a) Simplify the expression of $N$ as

$$
\begin{aligned}
N & =\left(F_{N}(K, N)-\bar{w}\right) N^{\prime}(\bar{w})+\frac{1}{\gamma} \sum_{e} \int e n(e, \bar{w}) u^{\prime}(c) p(a, \lambda, e) d a d \lambda \\
& +\sum_{e} \int e n(e, \bar{w}) u^{\prime \prime}(c)\left[q(1+\bar{r})-q^{\prime}\right] p(a, \lambda, e) d a d \lambda \\
& =\left(F_{N}(K, N)-\bar{w}\right) N^{\prime}(\bar{w})+\frac{1}{\gamma} \sum_{e} \int e n(e, \bar{w}) u^{\prime}(c) p_{q}(a, q, e) d a d q \\
& +\sum_{e} \int e n(e, \bar{w}) u^{\prime \prime}(c)\left[q(1+\bar{r})-q^{\prime}\right] p_{q}(a, q, e) d a d q \\
& =\left(F_{N}(K, N)-\bar{w}\right) N^{\prime}(\bar{w})+\frac{1}{\gamma} \sum_{e} \int e n(e, \bar{w}) u^{\prime}(c) m(a, e) d a \\
& +\sum_{e} \int e n(e, \bar{w}) u^{\prime \prime}(c)(1+\bar{r}) \mathbb{E}[q \mid a, e] m(a, e) d a \\
& -\sum_{e} \int e n(e, \bar{w}) u^{\prime \prime}(c) \mathbb{E}\left[q^{\prime} \mid a, e\right] m(a, e) d a .
\end{aligned}
$$

(b) If equation (39) holds, stop.

(c) If not, update $\bar{w}$.

i. If LHS of $(39)>$ RHS, adjust $\bar{w}$ down.

ii. If LHS $<$ RHS, adjust $\bar{w}$ up. 
iii. One potential new candidate of $\bar{w}$ can be computed as follows: use RHS of (39) to represent the new $N$ to backout the new $\bar{w}$ from (31).

6. Verify $T$ using (25).

\subsection{Transition Dynamics}

We consider three approaches to solve the transition dynamics. The first one is to guess the distribution of households on $a, \lambda, e$ and then update it. The second is to guess the price sequences and then to iterate on the price sequences, similar to how transition dynamics of Aiyagari model without taxes is solved. The last one is to use backward induction from the steady state. Let us first consider the transition dynamics without transfer.

\subsubsection{Guess the Distribution}

Main idea

- Guess the distribution $p_{t}\left(a_{t}, \lambda_{t}, e_{t}\right)$

- Solve from backward for policy functions and Lagrangian multipliers using the guessed $p_{t}$

- Compute forward the new distribution, starting from $p_{0}\left(a_{t}, \lambda_{t}, e_{t}\right)$

- Iterate until $p_{t}\left(a_{t}, \lambda_{t}, e_{t}\right)$ converge

Steps

1. Set all variables after $T$ at the steady state

2. Guess $\left\{p_{t}\left(a_{t}, \lambda_{t}, e_{t}\right)\right\}_{t=1}^{T-1}$

3. Starting from $t=T-1$, solve for variables at $t$ using variables from $t+1 \mathrm{on}$. We use guess and verify to find $\bar{w}_{t}$ and then other variables at $t$.

(a) Compute $\gamma_{t}$ from (8).

(b) Guess $\bar{w}_{t}$.

(c) Guess $\bar{r}_{t}$.

(d) Solve for $c_{t}=g_{c, t}\left(a_{t}, e_{t}\right)$ and $a_{t+1}=g_{a, t+1}\left(a_{t}, e_{t}\right)$ from (5) and (12). 
(e) Solve for $\lambda_{t+1}=g_{\lambda, t+1}\left(a_{t}, \lambda_{t}, e_{t}\right)$ from (7).

(f) Compute $A_{t}$ and $N_{t}$ from (15) and (16).

(g) Check whether $\bar{r}_{t}$ satisfies (10). Otherwise update on $\bar{r}_{t}$

(h) Solve for $K_{t}$ and $B_{t}$ from (13) and (14).

(i) Verify (11) and update $\bar{w}_{t}$.

i. If LHS of $(11)>$ RHS, reduce $\bar{w}_{t}$ as the new guess, and vise versa.

ii. A possible choice of the new guess of $\bar{w}_{t}$ is to use the RHS of (11) to get a new $N_{t}$ and then back out the new guess of $\bar{w}_{t}$ using (16).

4. Continue the backward induction until $t=0$.

5. Starting from $p_{0}\left(a_{0}, \lambda_{0}, e_{t}\right)$, compute the new $p_{t}\left(a_{t}, \lambda_{t}, e_{t}\right)$ using the new policy functions $a_{t+1}=g_{a, t+1}\left(a_{t}, e_{t}\right)$ and $\lambda_{t+1}=g_{\lambda, t+1}\left(a_{t}, \lambda_{t}, e_{t}\right)$.

6. Iterate until $p_{t}\left(a_{t}, \lambda_{t}, e_{t}\right)$ converge for each $t$.

\subsubsection{Guess the Price Sequences and Iterate on Them}

Main idea

- Guess $\left\{\bar{w}_{t}, \bar{r}_{t}\right\}_{t=0}^{\infty}$

- Solve the households' problem on $a_{t+1}\left(a_{t}, e_{t}\right)$ from backward

- Compute $m_{t}\left(a_{t}, e_{t}\right), A_{t}, B_{t}, K_{t}$ from forward

- Solve for $\gamma_{t}$ and $\lambda_{t+1}\left(a_{t}, e_{t}\right)$ from backward

- Compute $p_{t}\left(a_{t}, \lambda_{t}, e_{t}\right)$ from forward

- Check and update $\left\{\bar{w}_{t}, \bar{r}_{t}\right\}_{t=0}^{\infty}$

The steps are the following:

1. Guess $\left\{\bar{w}_{t}, \bar{r}_{t}\right\}_{t=0}^{\infty}$

2. Solve households' problem by backward induction, as in Aiyagari model

(a) Compute $n\left(e_{t}, \bar{w}_{t}\right)$ and $y\left(e_{t}, \bar{w}_{t}\right)$ 
(b) Solve for $a_{t+1}\left(a_{t}, e_{t}\right)$ by backward induction from from (5) and (12).

3. Compute $m_{t}\left(a_{t}, e_{t}\right)$, starting from $m_{0}\left(a_{0}, e_{0}\right)$ using (18) or simulation.

4. Compute $A_{t}$ and $N_{t}$ from (15) and (16).

5. Compute $K_{t}$ and $B_{t+1}$ forward using (14) and (13), namely,

$$
\begin{aligned}
K_{t} & =A_{t}-B_{t} \\
B_{t+1} & =F\left(K_{t}, N_{t}\right)-G_{t}+\left(1+\bar{r}_{t}\right) B_{t}+\bar{r}_{t} K_{t}+\bar{w}_{t} N_{t} .
\end{aligned}
$$

6. Compute $\gamma_{t}$ backward using

$$
\gamma_{t}=\beta\left(1+F_{K}\left(K_{t+1}, N_{t+1}\right)\right) \gamma_{t+1}
$$

7. Solve for $\lambda_{t+1}\left(a_{t}, \lambda_{t}, e_{t}\right)$ from

$$
\begin{aligned}
& u^{\prime}\left(c_{t}\right)+u^{\prime \prime}\left(c_{t}\right)\left[\lambda_{t}\left(1+\bar{r}_{t}\right)-\lambda_{t+1}\right] \\
& =\beta\left(1+\bar{r}_{t+1}\right) \mathbb{E}\left[u^{\prime}\left(c_{t+1}\right)+u^{\prime \prime}\left(c_{t+1}\right)\left[\lambda_{t+1}\left(1+\bar{r}_{t+1}\right)-\lambda_{t+2}\right] \mid e_{t+1}\right] \\
& +\beta \gamma_{t+1}\left(F_{K}\left(K_{t+1}, N_{t+1}\right)-\bar{r}_{t+1}\right) \\
& \text { if } a_{t+1}>-\underline{a}, \text { otherwise } \lambda_{t+1}=0 .
\end{aligned}
$$

Notice that $\lambda_{t+2}$ is a function of $\lambda_{t+1}$, namely, $\lambda_{t+2}=\lambda_{t+2}\left(a_{t+1}, \lambda_{t+1}, e_{t+1}\right)$.

8. Compute $p_{t}$ forward by simulaitons using $p_{0}$ and the policy functions: $a_{t+1}\left(a_{t}, e_{t}\right)$ and $\lambda_{t+1}\left(a_{t}, \lambda_{t}, e_{t}\right)$.

9. Check whether the guessed $\left\{\bar{w}_{t}, \bar{r}_{t}\right\}_{t=0}^{\infty}$ is the solution, and update if not.

(a) Check the equation

$$
\begin{aligned}
\gamma_{t} N_{t} & =\gamma_{t}\left(F_{N}\left(K_{t}, N_{t}\right)-\bar{w}_{t}\right) N^{\prime}\left(\bar{w}_{t}\right) \\
& +\sum_{e_{t}} \iint e_{t} n\left(e_{t}, \bar{w}_{t}\right)\left(u^{\prime}\left(c_{t}\right)+u^{\prime \prime}\left(c_{t}\right)\left[\lambda_{t}\left(1+\bar{r}_{t}\right)-\lambda_{t+1}\right]\right) .
\end{aligned}
$$


If it is not satisfied, update $\bar{w}_{t}$, for example by solving

$$
\begin{aligned}
\gamma_{t} N_{t}\left(\bar{w}_{t}^{\text {new }}\right) & =\gamma_{t}\left(F_{N}\left(K_{t}, N_{t}\right)-\bar{w}_{t}\right) N^{\prime}\left(\bar{w}_{t}\right) \\
& +\sum_{e_{t}} \iint e_{t} n\left(e_{t}, \bar{w}_{t}\right)\left(u^{\prime}\left(c_{t}\right)+u^{\prime \prime}\left(c_{t}\right)\left[\lambda_{t}\left(1+\bar{r}_{t}\right)-\lambda_{t+1}\right]\right) .
\end{aligned}
$$

(b) Check the equation

$$
\begin{aligned}
\gamma_{t} A_{t} & =\sum_{e_{t}} \iint u^{\prime}\left(c_{t}\right) \lambda_{t} p_{t}\left(a_{t}, \lambda_{t}, e_{t}\right) d a_{t} d \lambda_{t} \\
& +\sum_{e_{t}} \iint a_{t}\left(u^{\prime}\left(c_{t}\right)+u^{\prime \prime}\left(c_{t}\right)\left[\lambda_{t}\left(1+\bar{r}_{t}\right)-\lambda_{t+1}\right]\right) p\left(a_{t}, \lambda_{t}, e_{t}\right) d a_{t} d \lambda_{t}
\end{aligned}
$$

If it is not satisfied, update $\bar{r}_{t}$, for example by solving $\bar{r}_{t}^{\text {new }}$ from the above equation.

\subsubsection{Backward Induction}

Knowing the steady state, we can solve for the transition dynamics, given the initial bond $B_{0}$ and the initial asset and productivity distribution $m_{0}(a, e)$, by backward induction, with the following steps.

1. Set all variables After $T+1$ at the steady state levels.

2. At $T$, all variables except $B_{T}$ and $p_{T}$ are at the steady state levels. Guess $B_{T}$ and $p_{T}$.

3. Solve for variables at $t$ using variables from $t+1$ on. We use guess and verify to find $\bar{w}_{t}$ and then other variables at $t$.

(a) Compute $\gamma_{t}$ from (8).

(b) Guess $\bar{w}_{t}$.

(c) Guess $\bar{r}_{t}$.

(d) Solve for $c_{t}=g_{c, t}\left(a_{t}, e_{t}\right)$ and $a_{t+1}=g_{a, t+1}\left(a_{t}, e_{t}\right)$ from (5) and (12).

(e) Solve for $\lambda_{t+1}=g_{\lambda, t+1}\left(a_{t}, \lambda_{t}, e_{t}\right)$ from (7).

(f) Solve for $p_{t}\left(a_{t}, \lambda_{t}, e_{t}\right)$ from (17). One choice is to solve in two steps.

i. Solve for $m_{t}\left(a_{t}, e_{t}\right)$ from (18), using the knowledge of $g_{a, t+1}\left(a_{t}, e_{t}\right), \pi\left(e_{t+1} \mid e_{t}\right)$ and $m_{t+1}\left(a_{t+1}, e_{t+1}\right)$. 
ii. Solve for $p_{t}\left(\lambda_{t} \mid a_{t}, e_{t}\right)$ from (17), using the knowledge of $g_{\lambda, t+1}\left(a_{t}, \lambda_{t}, e_{t}\right), p_{t+1}\left(\lambda_{t+1} \mid a_{t+1}, e_{t+1}\right)$ and $m_{t}\left(a_{t}, e_{t}\right)$.

(g) Compute $A_{t}$ and $N_{t}$ from (15) and (16).

(h) Check whether $\bar{r}_{t}$ satisfies (10). Otherwise update on $\bar{r}_{t}$

(i) Solve for $K_{t}$ and $B_{t}$ from (13) and (14). We can substitute $B_{t}=A_{t}-K_{t}$ into (13) and get

$$
G_{t}+\left(1+\bar{r}_{t}\right) A_{t}-K_{t}+\bar{w} N_{t} \leq F\left(K_{t}, N_{t}\right)+B_{t+1}
$$

which leads to a unique solution of $K_{t}$.

(j) Verify (11) and update $\bar{w}_{t}$.

i. If LHS of $(11)>$ RHS, reduce $\bar{w}_{t}$ as the new guess, and vise versa.

ii. A possible choice of the new guess of $\bar{w}_{t}$ is to use the RHS of (11) to get a new $N_{t}$ and then back out the new guess of $\bar{w}_{t}$ using (16).

4. Continue the backward induction until $t=0$.

5. Check whether $p_{0}(\lambda, a, e)$ satisfies the initial conditions

(a) $m_{0}(a, e)=\int p_{0}(\lambda, a, e) d \lambda$ equals the initial asset and productivity distribution

(b) $p_{0}(\lambda \mid a, e)$ is degenerate distribution at $\lambda=0$

(c) If not, update the guess of $B_{T}$ and $p_{T}$. One possible way to get the new $B_{T}$ and $p_{T}$ is to compute the transition forward from the real $B_{0}$ and $p_{0}$, with the policy functions calculated. Then we will get a new sequence of $p_{t}$ and $B_{t}$, which give us a new guess of $p_{T}$ and $B_{T}$. 